\title{
LSTR AND ITS POTENTIAL ROLE IN CONSERVATIVE MANAGEIMENT OF NON-VITAL PRIMARY IMOLAR: A CASE REPORT.
}

KEYWORD: LSTR, NIET, 3-

Mix, 2-Mix, Primary Molars,

Chronic Irreversible Pulpitis, Non-vital Pulp.
Dr. Kapil Gandhi

Dr. Ritika Ahuja Malhotra

Dr. Sharon Jaiswal*

Dr. Shabana Alam

\section{Dr. Babita Jangra}

\section{Dr. Pratik Pande}

MDS, Professor and Head of Department of Pedodontics and Preventive Dentistry, Inderprastha Dental College and Hospital

(Reader) Department of Pedodontics and Preventive Dentistry, Inderprastha Dental College and Hospital

(Postgraduate student) Department of Pedodontics and Preventive Dentistry, Inderprastha Dental College and Hospital *Corresponding Author

(Postgraduate student) Department of Pedodontics and Preventive Dentistry, Inderprastha Dental College and Hospital

(Senior lecturer)- Department of Pedodontics and Preventive Dentistry, Inderprastha Dental College and Hospital

(Senior lecturer) Department of Pedodontics and Preventive Dentistry, Inderprastha Dental College and Hospital

E Lesion sterilization and tissue repair (LSTR) treatment is very effective in disinfecting the root canal using triple antibiotic mixed in a suitable vehicle. This report is highlighting a case where LSTR therapy was done using triple antibiotic paste in primary molar showing peri-radicular pathosis and root resorption. On one-year follow-up, the tooth H was asymptomatic with reduction in peri-radicular pathosis and root resorption. Thus, LSTR can be successful for nonvital pulp therapy in primary teeth with peri-radicular pathosis and root resorption. And also, LSTR therapy can be used as an alternative option to extraction in treating primary teeth with peri-radicular pathosis.

\section{INTRODUCTION}

Lesion Sterilization and Tissue Repair (LSTR) or NonInstrumental Endodontic Treatment (NIET) involves the use of three antibiotics/antibacterial drugs namely, Metronidazole, Ciprofloxacin and Minocycline (3Mix/Triple Antibiotic paste.). ${ }^{1}$ These antibacterial drugs help in eradicating bacteria in root canal and sterilize the root canal and periradicular region and results in good healing of the periradicular region. ${ }^{2}$ Deep layers of infected root canal wall consist of obligate anaerobes. Metronidazole has wide spectrum of bacterial action against oral obligate anaerobes, while Ciprofloxacin and Minocycline are added to sterilize the infected root dentin. ${ }^{3}$ Most commonly used vehicles are Propylene glycol and Macrogol which carry the 3-Mix into the entire dentin and help in killing all the bacteria in the lesions through the dentinal tubules. The cariology research unit at the Nigata University School of Dentistry had developed this concept in 1988.

Different combinations of antibiotics in the 3-Mix have been proposed till yet. There are concerns about staining with tetracycline-like drugs. Ruparel et al. in 2012 substituted Minocycline with Cefaclor in an in-vitro study which was referred as the modified Triple Antibiotic Paste and tried another combination referred to as the Double Antibiotic Paste (DAP). ${ }^{5}$ Sabrah et al. in 2013 found 2-Mix having comparable antibacterial activity to the 3-mix. ${ }^{6}$ Algarni et al. in 2015 used antibiotic gels containing Clindamycin in modified 3-Mix and compared it to 2-Mix and found 2-Mix having a similar bio-film inhibition as the modified 3-Mix consisting of Clindamycin. They also found 2-Mix to be more biocompatible than 3-Mix with Dental Pulp Stem Cells (DPSCs). ${ }^{7}$ This report highlighted the treatment outcome of LSTR done in primary molar tooth with peri-radicular pathosis using 2-Mix. (i.e., Metronidazole and Ciprofloxacin).

\section{CASE REPORT}

A 4-year-old child reported to the department of Pedodontics and Preventive Dentistry, with the chief complaint of pain and discomfort in his lower right back tooth region for 3 months.
Pain was spontaneous and aggravated on chewing food and at night. Clinical examination revealed a large carious lesion on the occlusal surface of the primary mandibular right second molar extending over to the lingual surface. The tooth showed normal physiologic mobility but was tender on percussion.

On intra-oral examination, there was deep occlusal caries i.r.t. 85. On radiographic examination, there was coronal radiolucency involving the enamel, dentine, pulp, and presence of internal resorption of the primary right mandibular second molar. Furcal radiolucency overlying the underlying succedaneous tooth was evident which was suggestive of chronic irreversible pulpitis. (Figure 1)

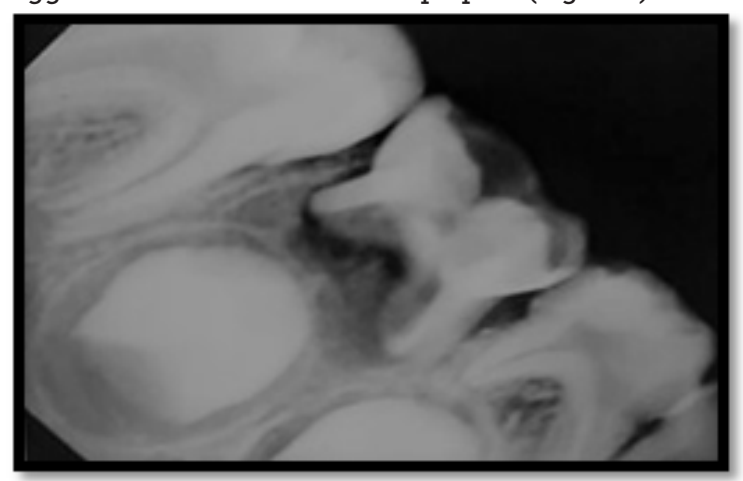

Figure 1. Pre-operative Radiograph I.r.t. 85

The child was extremely negative towards the dental extraction and parents did not consent for the treatment under sedation or general anesthesia for pharmacological behaviour management. Based on the clinical and radiographic findings, Lesion Sterilization and Tissue Repair was planned for concerned tooth. Access opening following extirpation of coronal and radicular pulps was done. Irrigation was done with sodium hypochlorite and saline.

\section{Dosage}

Ciprofloxacin (200mg), Metronidazole (500mg), Propylene 
glycol, Normal saline as carrier.

\section{Preparation OfThe 2-Mix}

- Ciprofloxacin tablet: after removing the enteric coating with a scalpel, the tablet was pulverized using sterilized mortar and pestle.

- Metronidazole:powder was segregated.

- The antibiotics were mixed together with propylene glycol in a ratio of $1: 1$.

- A creamy consistency was prepared.

Then this mix was rolled into a small ball of $1 \mathrm{~mm}$ diameter approximately and placed on pulpal floor and on the root canal orifices. This was followed by permanent restoration using glass ionomer cement. (Figure 2)

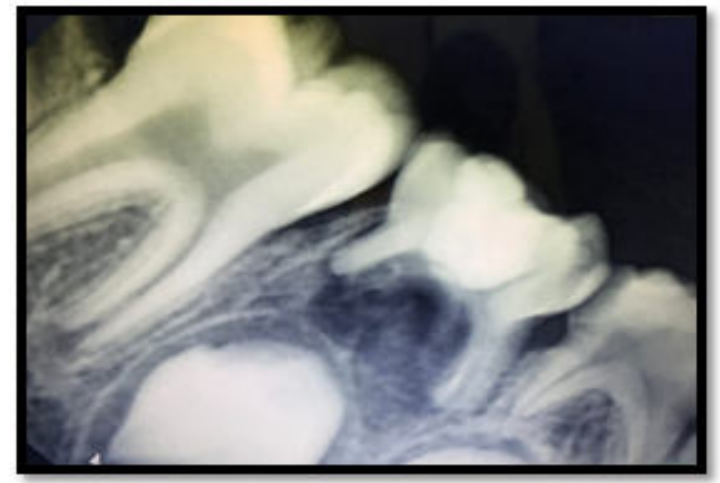

Figure. 2 Post-operative Periapical Radiograph.

Patient did not come for stainless steel crown on time, which was delayed for 6 months. The tooth was nontender on percussion and was asymptomatic. And stainless-steel crown placement was done i.r.t.85. (Figure 3)

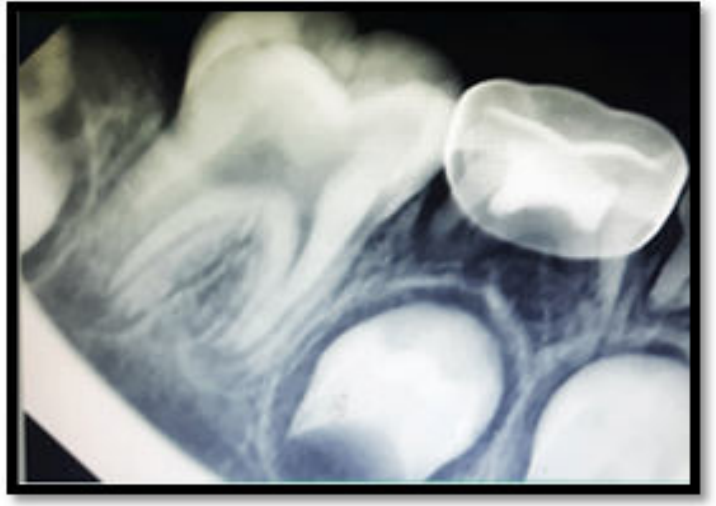

Figure 3. Stainless Steel Crown I.r.t. 85.

Upon 12 months follow-up, the tooth was asymptomatic with periapical healing (Figure 4). The child and his parents were satisfied as the child was able to chew food properly and there was no signs of pain or discomfort to the patient.

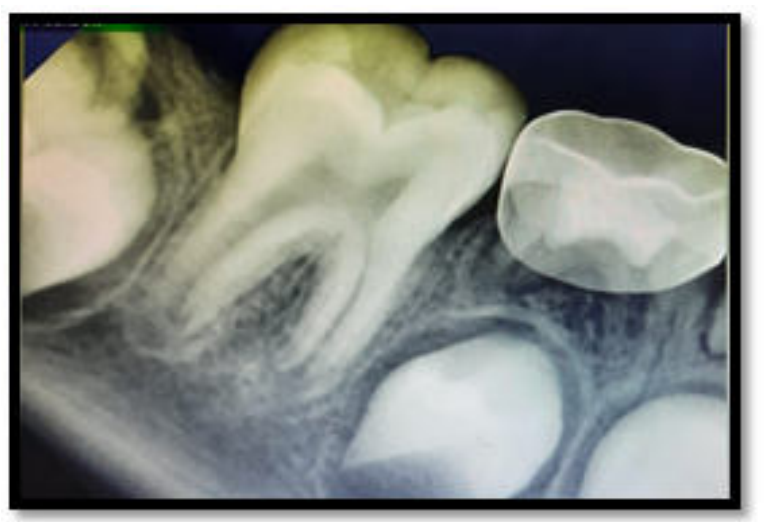

\section{DISCUSSION}

Lesion sterilization tissue repair treatment is indicated for primary teeth showing irreversible pulpitis or necrosis. Antibiotic mixtures used in LSTR for non-vital pulp therapies in primary teeth should not include tetracycline. ${ }^{8}$ This procedure is simple and minimally invasive, and helps in achieving maximum bacterial elimination from the root canal system without depending much on mechanical procedures. Also, preserving the primary tooth is the best space maintainer for the successor tooth.

In our case, the result after LSTR therapy in the molar tooth was encouraging. This therapy can be chosen for an excellent alternative to extractions and pulpectomies for non-vital primary teeth. The success of this treatment can be attributed to the bactericidal efficacy provided by the antibiotic mixture. In this case, 2-Mix was used instead of 3-Mix, which was done taking concern from various literature. ${ }^{6,7}$

2-Mix has its comparable antibacterial efficacy comparing to 3-Mix and also has longer residual antibacterial effect and fewer detrimental effects on DPSCs. It has similar mechanism of action i.e.; it sterilizes both pulpal and periapical infected tissues. In this case, main concern for localized Minocycline staining of the permanent tooth bud was also overcome, with the use of the 2-Mix. Also, this advocate for prudent and conservative use of antibiotics to minimize the risk of developing resistance to antibiotic regimens. ${ }^{4}$

Takushige et al showed normal physiologic root resorption utilizing LSTR therapy in case of primary teeth with periradicular lesions and also proper eruption of successor teeth without any disorders. ${ }^{9}$ In this regard LSTR can be a useful adjuvant to routine pulp therapies or alternate to pulpectomies in cases where child is uncooperative or canals are non-negotiable. LSTR therapy has revealed successful clinical results with the treatment of infected primary teeth in literature where success can be attributed to the bactericidal efficacy provided by the antibiotic mixture.

\section{CONCLUSION}

LSTR technique can be considered as an alternative treatment option for nonvital primary teeth, thus also avoiding a course of systemic antibiotics to the children. It is yet simple, timesaving and also cost-effective in relieving the symptoms. It has been proved to be a promising therapy in the treatment of primary molars exhibiting gross peri-radicular pathosis and internal resorption.

\section{REFERENCES:}

1. Sato, I., Ando-Kurihara, N., Kota, K., Iwaku, M., \& Hoshino, E. (1996). Sterilization of infected root-canal dentine by topical application of a mixture of ciprofloxacin, metronidazole and minocycline in situ. International endodontic journal, 29(2), 118-124

2. Taneja, S., \& Kumari, M. (2012). Use of triple antibiotic paste in the treatment of large periradicular lesions. Journal of investigative and clinical dentistry, 3(1), 72-76.

3. Hoshino, E., Iwaku, M., Sato, M. I. C. H. I. K. O., Ando, N., \& Kota, K. (1989). Bactericidal efficacy of metronidazole against bacteria of human carious dentin in vivo. Caries research, 23(2), 78-80.

4. Triveni, M. N., Dhaval, P., \& Rachappa, M. M. (2019). Lesion Sterilization and Tissue Repair (LSTR) Technique and its Clinical Application in Primary and Permanent Teeth:A Review.Ann Essence Dent, 11(4).

5. Ruparel,N.B.,Teixeira, F.B.,Ferraz, C.C.,\&Diogenes,A. (2012). Direct effect of intracanal medicaments on survival of stem cells of the apical papilla. Journal of endodontics, 38(10), 1372-1375.

6. Sabrah, A. H., Yassen, G. H., \& Gregory, R. L. (2013). Effectiveness of antibiotic medicaments against biofilm formation of Enterococcus faecalis and Porphyromonas gingivalis. Journal of endodontics, 39(11), 1385-1389.

7. Algarni, A. A., Yassen, G. H., \& Gregory, R. L. (2015). Inhibitory effect of gels loaded with a low concentration of antibiotics against biofilm formation by Enterococcus faecalis and Porphyromonas gingivalis. Journal of Oral Science, 57(3), 213-218.

8. American Academy of Pediatric Dentistry. (2020) Pulp therapy for primary and immature permanent teeth. The Reference Manual of Pediatric Dentistry. Chicago, Ill.: American Academy of Pediatric Dentistry, 384-92.

9. Takushige, T., Cruz, E. V., Asgor Moral, A., \& Hoshino, E. (2004). Endodontic treatment of primary teeth using a combination of antibacterial drugs. International endodontic journal,37(2), 132-138.

Figure 4. Radiograph After 12 Months Follow-up. 